\title{
DIRECT EFFECTS OF QUERCETIN ON IMPAIRED REACTIVITY OF SPONTANEOUSLY HYPERTENSIVE RAT AORTAE: COMPARATIVE STUDY WITH ASCORBIC ACID
}

\author{
Machha Ajay,* Francis I Achike, ${ }^{\dagger}$ Ali Mohd Mustafa,* and Mohd Rais Mustafa* \\ *Department of Pharmacology, Faculty of Medicine, University of Malaya and ${ }^{\dagger}$ International Medical University, Kuala \\ Lumpur, Malaysia
}

\section{SUMMARY}

1. There is a growing interest in the anti-oxidant characteristics and use of flavonoids in the management of cardiovascular diseases. The cardiovascular mechanism of action of these plant derivatives remains controversial. This study compared the effects of the flavonoid quercetin with those of the anti-oxidant vitamin ascorbic acid (vitamin $\mathrm{C}$ ) on the reactivity of aortic rings from spontaneously hypertensive rats (SHR).

2. The phenylephrine (PE)-induced contractile and the endothelium-dependent and independent relaxant responses of aortic rings from 21 to 22 week old SHR and age-matched normotensive Wistar (WKY) rats were observed in the presence of quercetin or ascorbic acid. All the experiments were performed in the presence of the cyclooxygenase inhibitor, indomethacin (10 $\mu \mathrm{mol} / \mathrm{L})$.

3. The endothelium-dependent and independent relaxations to acetylcholine (ACh) and sodium nitroprusside (SNP), respectively, were significantly lesser in the SHR compared to the WKY tissues whereas the contractile responses to $P E$ were similar in both tissues. Pretreatment of WKY rings with quercetin or ascorbic acid had no effect on the responses to ACh or PE. In the SHR tissues, however, quercetin or ascorbic acid significantly improved the relaxation responses to $\mathrm{ACh}$ and reduced the contractions to $\mathbf{P E}$ with greater potency for quercetin. Both compounds lacked any effects on the responses to SNP in either aortic ring types. N⿴-nitro-L-arginine methyl ester (L-NAME, $10 \mu \mathrm{mol} / \mathrm{L}$ ) significantly attenuated the vasodepressor effects of quercetin and ascorbic acid, raising the responses to $\mathrm{PE}$ to a level similar to that observed in the control SHR tissues. In L-NAME pretreated aortic rings, quercetin and ascorbic acid inhibited the contractile responses to PE with the same magnitude in WKY and SHR tissues.

4. The present results suggest that acute exposure to quercetin improves endothelium-dependent relaxation and reduces the contractile responses of hypertensive aortae with a greater potency than ascorbic acid. This suggests a better vascular

Correspondence: Dr Mohd Rais Mustafa, Department of Pharmacology, Faculty of Medicine, University of Malaya, Kuala Lumpur, 50603, Malaysia. Email: rais@um.edu.my

Received 16 August 2005; revision 30 October 2005; accepted 13 November 2005.

CC 2006 Blackwell Publishing Asia Pty Ltd protection with this flavonoid than ascorbic acid in the SHR model of hypertension and possibly in human cardiovascular diseases.

Key words: anti-oxidants, ascorbic acid, endothelium, hypertension, nitric oxide, quercetin, SHR.

\section{INTRODUCTION}

Hypertension is one of the common risk factors for the development of coronary artery disease (CAD). It is associated with oxidative stress and increased free radicals, which may contribute to the development of complications such as the reduction in endothelium-dependent relaxation of vascular smooth muscles. ${ }^{1,2}$ Superoxide anion is an important member of the free radical family and together with its derivatives, it counteracts the relaxing activity of endothelium-derived nitric oxide (EDNO) and prostacyclin $\left(\mathrm{PGI}_{2}\right){ }^{3,4}$ Prevailing evidence from both human and animal studies indicate that dietary anti-oxidants can prevent the development of the vascular complications associated with hypertension., ${ }^{5,6}$

Recent evidence suggests flavonoids, a group of polyphenolic compounds found in human diet, as potential candidates for the prevention of morbidity and mortality due to CAD. ${ }^{7,8}$ The vascular beneficial effects of flavonoids are attributed primarily to their interactions with the EDNO system. ${ }^{9}$ Flavonoids have been shown to improve the bioavailability of EDNO by directly interacting with superoxide anions and other free radicals which are known to destroy EDNO ${ }^{10,11}$ In addition to enhancing EDNO bioavailability, flavonoids are also reported to produce relaxant effects in various vascular preparations by enhancing EDNO production either by stimulating the activity or by enhancing the expression of endothelial nitric oxide synthase (eNOS) ${ }^{12-14}$ Quercetin is the most commonly found flavonoid in the human diet and has been shown to possess potent vasodilator and anti-oxidant activities. ${ }^{13,15,16}$ Indeed, chronic oral treatment with quercetin has been reported to protect against impaired vascular endothelial function in various models of experimental hypertension. ${ }^{17,18}$ Unfortunately, there was only one study looking at the acute effects of quercetin on blunted endothelium-dependent relaxations in isolated hypertensive arteries. ${ }^{19}$ Furthermore, to our knowledge, no studies have compared the endothelial protective effects of flavonoids with those of dietary anti-oxidant vitamins. Therefore, the present study examined the direct effects of quercetin on endothelium-dependent relaxation induced by acetylcholine $(\mathrm{ACh})$ in isolated aortic rings from spontaneously hypertensive rats (SHR). The effects of quercetin on the responsiveness of SHR aortic rings to endothelium-independent vasodilator sodium nitroprusside (SNP) or to $\alpha_{1}$-adrenergic receptor 
agonist phenylephrine (PE) were also examined. The effects of quercetin were compared with those of L-ascorbic acid (vitamin C).

\section{MATERIALS AND METHODS}

\section{Drugs and chemicals}

L-ascorbic acid, quercetin, phenylephrine- $\mathrm{HCl}$, acetylcholine chloride, indomethacin and $\mathrm{N} \omega$-nitro-L-arginine methyl ester were purchased from Sigma Chemicals (St. Louis, MO, USA). Sodium nitroprusside and Kreb's salts were purchased from BDH Limited (Poole, England). Except for indomethacin, quercetin and ascorbic acid all the other drug solutions were prepared freshly on the day of experiment by dissolving weighed amount of respective drugs in distilled water. Indomethacin was prepared in stock $(10 \mathrm{mmol} / \mathrm{L})$ in $0.5 \%$ w/v sodium carbonate and diluted with distilled water. Quercetin and ascorbic acid were each prepared as stock solution, $10 \mathrm{mmol} / \mathrm{L}$, with dimethyl sulfoxide (DMSO). The final concentrations were prepared by serial dilutions with distilled water; DMSO concentration was adjusted to less than $0.05 \% \mathrm{v} / \mathrm{v}$.

\begin{abstract}
Animals
The experiments were performed on male spontaneously hypertensive rats (SHR) and their sex- and age-matched normotensive Wistar-Kyoto (WKY) rats weighing 250-300 g (21-22 weeks old). The animals were housed in plastic cages (four in each) with free access to standard rat chow (Gold Coin Sdn. Bhd. Malaysia) and tap water. All the experimental procedures were subjected to The University of Malaya Animal Experimentation Ethics Committee approval.
\end{abstract}

\section{Vascular ring preparation}

The animals were anaesthetized with a single intraperitoneal dose of pentobarbitone sodium $(60 \mathrm{mg} / \mathrm{kg}$ bodyweight). Thereafter, the descending thoracic aorta was excised through a ventral incision and was cleaned of fat and connective tissues; care was taken not to dislodge the vascular endothelium. The aorta was sectioned into small rings (3-5 $\mathrm{mm}$ in width), which were then mounted in a jacketed organ bath containing $5 \mathrm{~mL}$ of normal Kreb's physiological salt solution (KPSS) of the following composition (mmol/L): $\mathrm{NaCl}$ 118.2, $\mathrm{KCl} 4.7, \mathrm{CaCl}_{2} .2 \mathrm{H}_{2} \mathrm{O}$ 2.5, $\mathrm{KH}_{2} \mathrm{PO} 41.2, \mathrm{MgCl}_{2}$ 1.2, glucose 11.7, $\mathrm{NaHCO}_{3} 25.0$, and EDTA 0,026 . The bath solution was maintained at $37^{\circ} \mathrm{C}$ and oxygenated continuously with a mixture of $95 \%$ oxygen and $5 \%$ carbon dioxide. The rings were attached to isometric force-displacement transducers (Grass Instrument Co. Quincy, MA) and the transducer output was amplified and recorded continuously using the Mac Laboratory recording system (AD Instruments, Australia) connected to a portable computer display monitor. The rings were equilibrated for $45 \mathrm{~min}$ under $1 \mathrm{~g}$ resting tension. During this period of stabilization the bath solution was replaced every $15 \mathrm{~min}$. Following the equilibration, the contractile responses of aortic rings were tested for viability by the addition of KPSS containing $10 \%(\mathrm{w} / \mathrm{v}) \mathrm{KCl}\left(\right.$ high $\mathrm{K}^{+}$). The rings were contracted for 5 min every 10 min until two consecutive equal contractions were attained - evidence of tissue stability. Following washout of the responses to high $\mathrm{K}^{+}$, the rings were incubated with indomethacin $(10 \mu \mathrm{mol} / \mathrm{L})$ for $10 \mathrm{~min}$ and further experimental procedures were carried out in its presence. The concentration of quercetin was selected based on previous investigations from this lab which showed that at this concentration quercetin produced more than $50 \%$ relaxation in high $\mathrm{K}^{+}$and PE precontracted aortic rings from WKY rats. ${ }^{13}$

\section{Pharmacological studies}

The responses of aortic rings from SHR and WKY rats to endotheliumdependent and -independent relaxants ACh and SNP, respectively, were examined under submaximal PE $(10 \mu \mathrm{mol} / \mathrm{L})$-induced contraction. The responses of the aortic rings to cumulative concentrations of $\mathrm{ACh}$ (from $10^{-10}$ to $10^{-5} \mathrm{~mol}$ ) or SNP (from $10^{-11}$ to $10^{-6} \mathrm{~mol}$ ) were recorded at intervals of
3 min in separate tissues incubated in KPSS to which was added vehicle, quercetin $(10 \mu \mathrm{mol} / \mathrm{L})$ or ascorbic acid $(10 \mu \mathrm{mol} / \mathrm{L}) 20 \mathrm{~min}$ prior to $\mathrm{PE}$ stimulation and throughout the experiment.

In another set of experiments, aortic rings from SHR or WKY rats were incubated for $20 \mathrm{~min}$ in KPSS to which was added vehicle, quercetin $(10 \mu \mathrm{mol} / \mathrm{L})$ or ascorbic acid $(10 \mu \mathrm{mol} / \mathrm{L})$ and the contractile responses to cumulative concentrations of PE (from $10^{-10}$ to $10^{-5} \mathrm{~mol}$ ) were recorded at intervals of $3 \mathrm{~min}$. The protocol was repeated with a separate set of aortic rings which were exposed, throughout the experiment, to the eNOS inhibitor $\omega$-nitro-L-arginine methyl ester (L-NAME, $10 \mu \mathrm{mol} / \mathrm{L}$ ).

\section{Data presentation and statistical analysis}

The concentrations given in the text or in figures represent final bath concentrations of respective agonists. The relaxant responses to $\mathrm{ACh}$ and SNP were calculated as percentage reduction of the PE $(1 \mu \mathrm{mol} / \mathrm{L})$-induced maximal contraction, while the contractile responses to PE were recorded as percentage of the maximum contractile response to high $\mathrm{K}^{+}$. With the aid of a computer software (Prism 2.0, Graph Pad software, USA) the values were plotted to obtain a best-fit dose-response curve with the maximal response $\left(\mathrm{R}_{\max }\right.$, maximum agonist-induced response) and $\mathrm{pEC}_{50}$ (negative logarithm of $50 \%$ effective concentration) values determined. The observed responses were analysed for statistical significance using Student's $t$-test (Microsoft Excel, Microsoft Corp., USA) for unpaired observations and the one way analysis of variance (ANOVA) for multiple value comparison (Prism 2.0, GraphPad Software, USA). A value of $P<0.05$ was considered statistically significant.

\section{RESULTS}

\section{ACh- and SNP-induced vasodilatations}

Figure 1 summarizes the data on the relaxant effects of ACh in the different experimental conditions. The cumulative addition of ACh led to a concentration-dependent relaxation of PE-contracted WKY and SHR aortae (Fig. 1). Control SHR aortic rings demonstrated marked reduction in their response to ACh compared to rings from control WKY rats (Fig. 1/ Table 1). In WKY aortic rings incubated either with quercetin or ascorbic acid their peak relaxation responses to ACh remain comparable to control WKY aortae (Table 1). In SHR aortic rings incubated with quercetin $(10 \mu \mathrm{mol} / \mathrm{L})$ the peak relaxant effect to ACh was markedly improved compared to control SHR aortic rings (Table 1). Indeed, the relaxations to ACh were restored to a similar extent as in control WKY aortae (Fig. 1). Similarly, in SHR aortic rings incubated with L-ascorbic acid the maximal response to ACh was significantly improved (Fig. 1/Table 1).

Figure 2 illustrates the relaxant responses to endotheliumindependent vasodilator, SNP, in all experimental groups. The maximal relaxant response to SNP was significantly lesser in control SHR aortic rings compared with aortic rings attained from WKY rats (Fig. 2/ Table 1). In addition, irrespective of the quercetin or ascorbic acid pretreatment, the vasodilatation responses to SNP remain unchanged in both WKY and SHR aortae compared to their respective controls (Fig. 2/Table 1).

\section{Contractile responses to $\mathrm{PE}$ stimulation}

The exposure of vehicle-treated (control) WKY and SHR aortic rings to $\mathrm{PE}$ led to a concentration-dependent rise in tension in both experimental groups (Fig. 3). There were no significant differences in the responses to PE between the WKY and SHR aortic rings (Fig. 3/Table 1). In WKY aortic rings incubated either with quercetin 


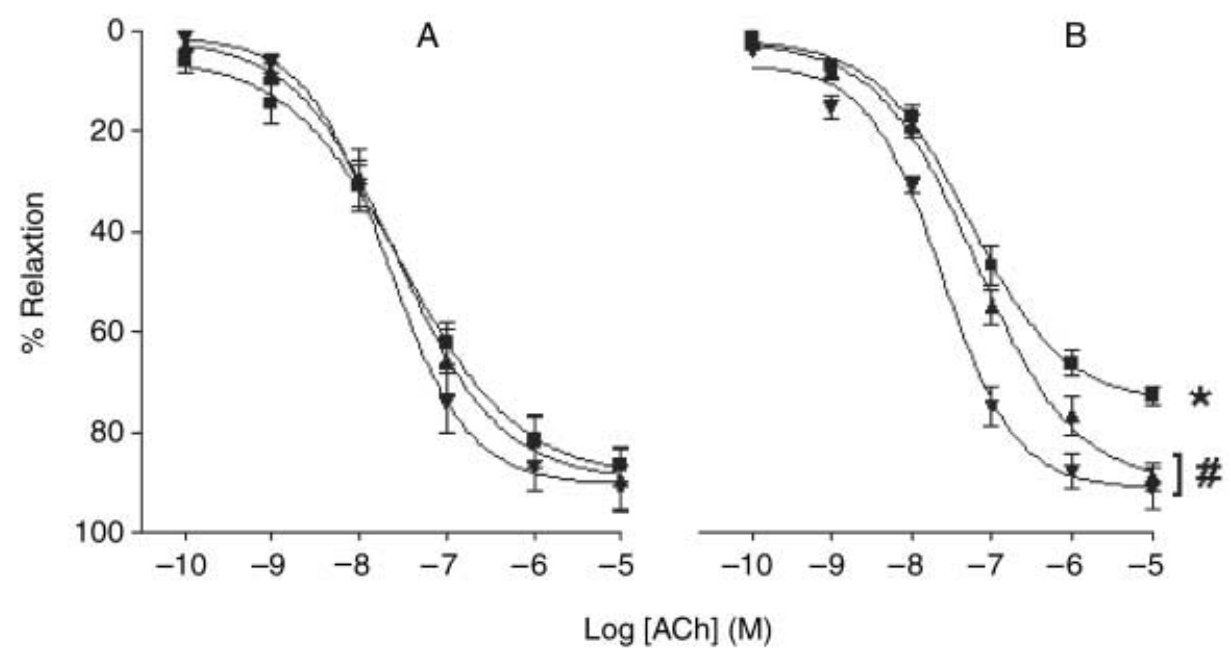

Fig. 1 Relaxant responses to endotheliumdependent vasodilator acetylcholine (ACh) in vehicle (control, $\mathbf{\square})$, quercetin $(\boldsymbol{\nabla})$ or ascorbic acid $(\mathbf{\Delta})$ incubated aortic rings from WKY (A) and SHR (B) rats. Symbols represent mean \pm SEM of 6 or 7 experiments. ${ }^{*} P<0.05$ control WKY versus control SHR aortic rings, ${ }^{\#} P<0.05$ control SHR versus quercetin or ascorbic acid incubated SHR aortic rings.

Table 1 Responses of normal (WKY) and hypertensive (SHR) aortic rings to endothelium-dependent vasodilator acetylcholine (ACh), to endotheliumindependent vasodilator sodium nitroprusside (SNP) and to $\alpha_{1}$-adrenergic receptor agonist phenylephrine (PE) under different experimental conditions. Values represent mean \pm SEM of 6 or 7 experiments

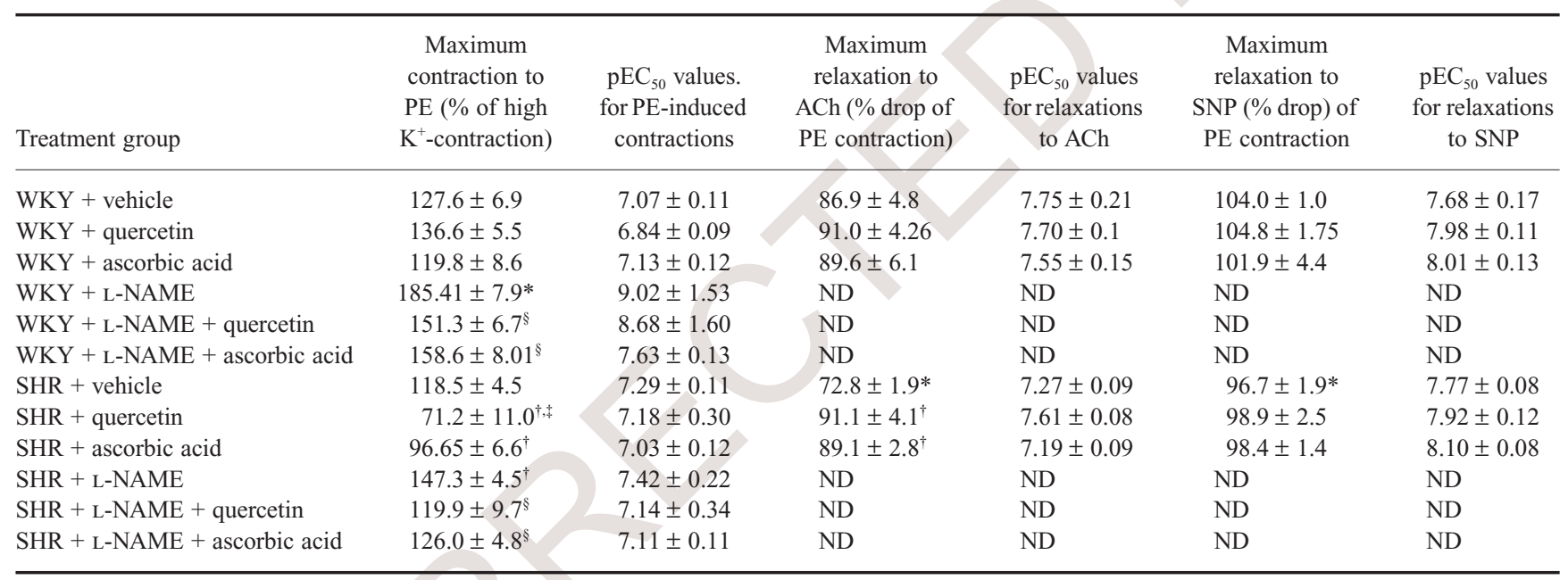

$\mathrm{ND}=$ not determined, ${ }^{*} P<0.05$ versus control WKY aortic rings, ${ }^{\dagger} P<0.05$ versus control SHR aortic rings, ${ }^{\ddagger} P<0.05$ versus ascorbic acid incubated SHR aortic rings, ${ }^{\S} P<0.05$ versus corresponding L-NAME pretreated aortic rings.

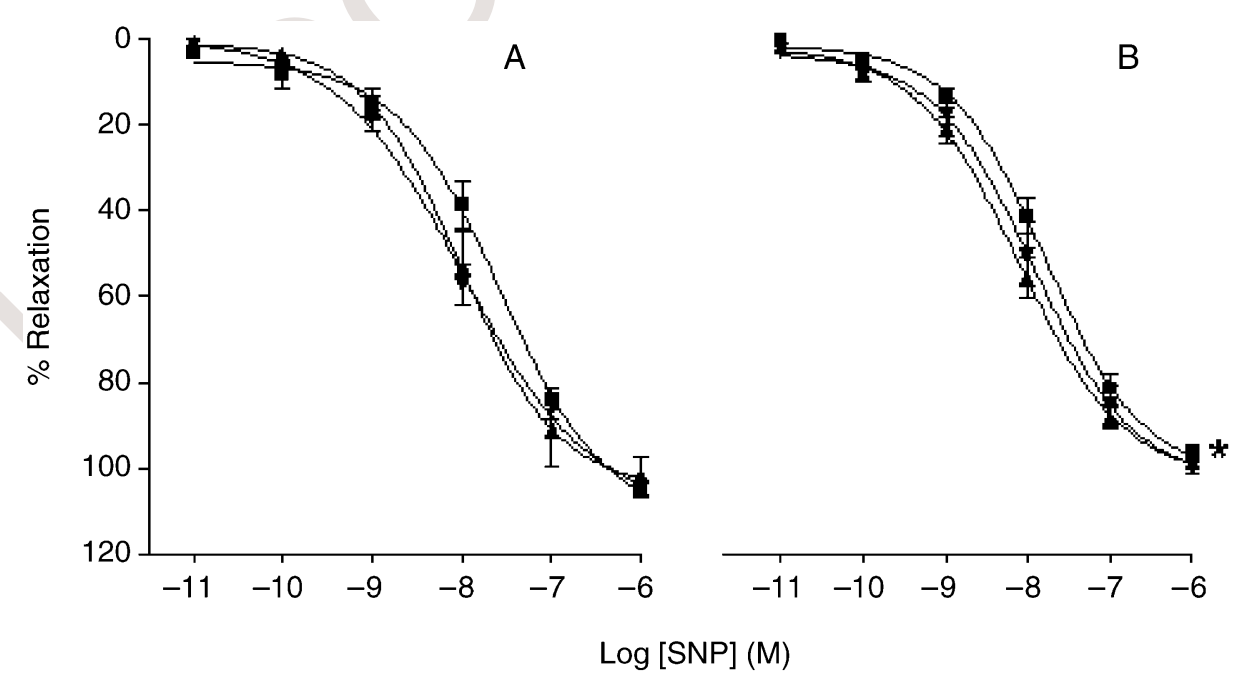

Fig. 2 Relaxant responses to endotheliumindependent vasodilator sodium nitroprusside (SNP) in vehicle (control, $\mathbf{\square}$ ), quercetin $(\boldsymbol{\nabla})$ or ascorbic acid $(\mathbf{\Delta})$ incubated aortic rings from WKY (A) and SHR (B) rats. Symbols represent mean \pm SEM of 6 or 7 experiments. ${ }^{*} P<0.05$ control WKY versus control SHR aortic rings. 
Fig. 3 Contractile responses to PE in vehicle (control, $\mathbf{\square})$, quercetin $(\boldsymbol{\nabla})$ or ascorbic acid $(\mathbf{\Delta})$ pretreated aortic rings from WKY (A) and SHR (B) rats. Symbols represent mean $+\mathrm{SEM}$ of 6 or 7 experiments. $* P<0.05$ control SHR versus quercetin or ascorbic acid pretreated SHR aortic rings, ${ }^{\sharp} P<0.05$ quercetin versus ascorbic acid pretreated SHR aortic rings.

Fig. 4 PE-induced contractions of WKY (A) and SHR (B) aortic rings pretreated with vehicle (Control, $\square)$, L-NAME $(\diamond)$, L-NAME with quercetin $(\boldsymbol{\nabla})$ or L-NAME with ascorbic acid $(\mathbf{\Lambda})$. Symbols represent mean \pm SEM of 6 or 7 experiments. $* P<0.05$ control versus L-NAME pretreated aortic rings, ${ }^{\sharp} P<0.05$ L-NAME versus L-NAME with quercetin or L-NAME with ascorbic acid pretreated aortic rings.


or ascorbic acid the peak contractile response to PE remained comparable $(P>0.05)$ to the control WKY aortae (Table 1/Fig. 3 ). In contrast, in SHR aortic rings incubated either with quercetin or ascorbic acid the peak contractile response to PE was significantly lesser than that of the control SHR aortic rings (Fig. 3/Table 1). In addition, compared with ascorbic acid- incubated SHR aortic rings, the maximal contractile response to PE was significantly lesser in the quercetin- incubated SHR tissues (Table 1).

In the presence of $\mathrm{L}-\mathrm{NAME}(10 \mu \mathrm{mol} / \mathrm{L})$ the maximum contractile response of WKY and SHR aortic rings to PE was significantly enhanced (Table 1). This enhanced contraction was however, partially reversed by quercetin and ascorbic acid in WKY, but not SHR tissues where the L-NAME effect was fully reversed (Table 1/ Fig. 4). In addition, in the presence of L-NAME the peak contractile responses to PE of the quercetin- or ascorbic acid- treated SHR aortic rings were essentially similar to that in control SHR aortic rings (Table 1/Fig. 4).

\section{DISCUSSION}

Consistent with earlier reports of impaired $\mathrm{ACh}$-induced relaxation in hypertension, ${ }^{1,2,5,6}$ the relaxation responses to $\mathrm{ACh}$ were significantly lesser in hypertensive aortae compared to normotensive aortae (Fig. 1). The incubation of the hypertensive tissues with either quercetin or ascorbic acid enhanced their relaxation responses to ACh indicating that ascorbic acid and quercetin promote the EDNO-mediated relaxant response to ACh in the SHR tissues. Blunted ACh-induced relaxation in hypertensive arteries have been previously attributed to overproduction of endothelium-derived constrictor prostanoids and/or superoxide anions. ${ }^{3,4,20}$ All experiments in this study were performed in the presence of the cyclooxygenase inhibitor, indomethacin, with the view to eliminating the generation of constrictor or relaxant prostanoids. The blunted relaxations to ACh observed in hypertensive tissues therefore are probably the result of the detrimental effects of superoxide anions and other free radicals on EDNO and not the result of EDNO-prostanoid cross-talk. It follows that the enhancement by quercetin or ascorbic acid of the ACh-induced relaxation in hypertensive tissues is probably due to its superoxide anion scavenging property as previously reported. ${ }^{10,21}$ A superoxide scavenging role for quercetin or ascorbic acid is also consistent with its inhibition of PE-induced contraction of hypertensive tissues (Fig. 3). This inhibition, as argued above, cannot be attributed to a cyclooxygenase product. We suggest that by scavenging superoxide anions (anti-oxidant effect) quercetin or ascorbic acid enhanced the bioavailability of EDNO, thus, enhancing the relaxant responses of hypertensive tissues to ACh (Fig. 1) or inhibiting their contractile responses to PE (Fig. 3). The inhibitory effects of quercetin or ascorbic acid on PE-induced contraction of hypertensive aortas (Fig. 3) were significantly prevented in the absence of EDNO 
synthesis (i.e. presence of L-NAME- Fig. 4), again suggesting that the effects of quercetin and ascorbic acid were mediated, at least in part, by improved EDNO.

Interestingly, the (ACh) relaxation-enhancing and (PE) contractioninhibiting effects of quercetin and ascorbic acid on hypertensive (SHR) tissues were not observed with the normal (WKY) tissues. It is well known that normotensive WKY tissues produce little or no free radicals. ${ }^{4,21}$ We therefore postulate that the failure to elicit effect with quercetin or ascorbic acid in the normal tissues is a reflection of the absence of free radicals in those tissues. The direct measurement of free radicals which we were unable to perform in this study will be useful in clarifying these assumptions.

Incubation of either normal or hypertensive tissues with quercetin or ascorbic acid did not enhance the relaxant responses to the endothelium-independent vasodilator, SNP. Perhaps by enhancing NO bioavailability through its NO donor effect, SNP provided full NO-mediated relaxation, thus 'usurping' the action of quercetin and ascorbic acid. This view is consistent with reports by earlier workers that flavonoids accumulate on the surface between the endothelial and vascular smooth muscle cells $\mathrm{s}^{10,22}$ and exert beneficial vascular effects by either enhancing EDNO production or inhibiting its destruction by free radicals; in either case improving EDNO bioavailability. ${ }^{10-14}$

Defects in the nitric oxide signal transduction pathways including the down regulation of guanylate cyclase (sGC) followed by impaired cGMP production are the other possible mechanisms implicated in the impaired responses of hypertensive vessels. ${ }^{23,24}$ This view is supported by the observed reduction in $\mathrm{SNP} / \mathrm{NO} / \mathrm{sGC} /$ cGMP-mediated relaxation responses in hypertensive compared to the normotensive aortae (Table 1/Fig. 2), given that in both cases NO bioavailability has been assured by the NO donor- SNP. Treatment of the hypertensive vessels with quercetin or ascorbic acid did not enhance the relaxant response to SNP. This is an indirect evidence that the beneficial (relaxant) effect of quercetin or ascorbic acid is not through the enhancement of the downstream (NO/sGC/cGMP) NO signal transduction pathway, but rather by improving the upstream EDNO bioavailability as described above.

Quercetin has been shown in vitro to possess higher superoxide scavenging and anti-oxidant activities than ascorbic acid. ${ }^{15,25}$ Consistent with these reports, quercetin in the present study elicited a significantly higher reduction in the constrictor responses to PE than did ascorbic acid in hypertensive tissues (Fig. 3). In addition, quercetin also elicited a greater enhancement of $\mathrm{ACh}$-induced relaxation of hypertensive tissues (Fig. 1).

In normotensive (WKY) tissues, treatment with L-NAME caused significant increases in the contractile responses to PE (Fig. 4) which was partially reversed by incubation of the tissues in either quercetin or ascorbic acid. Granted that the L-NAME-induced increased contraction is a result of abolition of EDNO production, it could be argued that the partial reversal of the L-NAME effect is an indication that quercetin and ascorbic acid probably inhibit PE-induced contraction by some L-NAME-insensitive process. This, as previously reported for some flavonoids, may be an L-NAME insensitive NO pool or endothelium-derived hyperpolarising factor. ${ }^{26,27}$ In the SHR tissues both quercetin and ascorbic acid reversed the L-NAME increased contraction to normal level. This result is consistent with the view that the anti-oxidants (ascorbic acid or quercetin) inhibit PEinduced contraction in the hypertensive tissues through enhancing an L-NAME sensitive NO pool. The complete reversal of the contraction in SHR as against WKY tissues suggests that the L-NAME insensitive mechanisms observed in the WKY may be down-regulated or absent in the SHR tissues.

In conclusion, the results showed that acute exposure to quercetin improves endothelium-dependent vascular relaxation and reduces $\alpha_{1}$-adrenergic receptor mediated contractions of isolated hypertensive aortae, with greater potency than ascorbic acid. This suggests a better vascular protective effect with this flavonoid than with ascorbic acid in SHR model of hypertension and possibly in human cardiovascular diseases. These effects of quercetin probably results from its endothelial nitric oxide protective effects although participation of other mechanism(s) can not be ruled out.

\section{ACKNOWLEDGEMENTS}

This study was supported by an IRPA grant (No: 06-02-03-6020) from Ministry of Science, Technology and Innovation, Malaysia.

\section{REFERENCES}

1. Lassegue B, Griendling KK. Reactive oxygen species in hypertension. Am. J. Hypertens 2004; 17: 852-60.

2. Berry C, Brosnan MJ, Fennell J, Hamilton CA, Dominiczak AF. Oxidative stress and vascular damage in hypertension. Curr. Opin. Nephrol. Hypertens 2001; 10: 247-55.

3. Cuzzocrea S, Mazzon E, Dugo L, Di Paola R, Caputi AP, Salvemini D. Superoxide: A key player in hypertension. FASEB. J. 2004; 18 (1): 94-101.

4. Katusic ZS. Superoxide anion and endothelial regulation of arterial tone. Free. Radic. Biol. Med. 1996; 20 (3): 443-8.

5. Taddei S, Virdis A, Ghiadoni L, Magagna A, Salvetti A. Vitamin C improves endothelium-dependent vasodilation by restoring nitric oxide activity in essential hypertension. Circulation 1998; 97: 2222-9.

6. Rodriguez-Porcel M, Herrman J, Chade AR et al. Long-term antioxidant intervention improves myocardial microvascular function in experimental hypertension. Hypertension 2004; 43 (2): 493-8.

7. Hertog MGL, Feskens EJM, Hollman PCH, Katan MB, Kromhurt D. Dietary antioxidant flavonoids and risk of coronary heart disease: The Zutphen Elderly Study. Lancet 1993; 342: 1007-11.

8. Knekt P, Jarvinen R, Reunanen A, Maatela J. Flavonoid intake and coronary mortality in Finland: A cohort study. Br. Med. J. 1996; 312: $478-81$.

9. Cook NC, Samman S. Flavonoids- Chemistry, metabolism, cardioprotective effects, and dietary sources. Nutr. Biochem. 1996; 7: 66-76.

10. Heijnen CGM, Haenen GRMM, Wiseman SA, Tijburg LBM, Bast A. The interaction of tea flavonoids with the NO-system: Discrimination between good and bad. Food Chem. 2000; 70: 365-70.

11. Nijveldt RJ, van Nood E, van Hoorn DE, Boelens PG, van Norren K, van Leeuwen PA. Flavonoids: A review of probable mechanisms of action and potential applications. Am. J. Clin. Nutr 2001; 74: $418-25$.

12. Lorenz M, Wessler S, Follman E et al. A constituent of green tea, apigallocatechin-3-gallate, activates endothelial nitric oxide synthase by a phosphatidylinositol-3-OH-kinase-, cAMP-dependent protein kinase, and Akt-dependent pathway and leads to endothelial-dependent vasorelaxation. J. Biol. Chem. 2004; 279: 6190-5.

13. Ajay M, Gilani AU, Mustafa MR. Effects of flavonoids on vascular smooth muscle of the isolated rat thoracic aorta. Life Sci. 2003; 74 : 603-12.

14. Wallerath T, Poleo D, Li H, Fostermann U. Red wine increases the expression of human endothelial nitric oxide synthase: A mechanism that may contribute to its beneficial cardiovascular effects. J. Am. Coll. Cardiol. 2003; 41: 471-8.

15. Kaneyuki T, Noda Y, Traber MG, Mori A, Packer L. Superoxide anion and hydroxyl radical scavenging activities of vegetable extracts 
measured using electron spin resonance. Biochem. Mol. Biol. Int. 1999; 47 (6): 979-89.

16. Rice-Evans CA, Miller NJ, Paganga G. Structure-antioxidant activity relationships of flavonoids and phenolic acids. Free. Radic. Biol. Med. 1996; 20: 933-56.

17. Galisteo M, Garcia-Saura MF, Jimenez R et al. Effects of quercetin treatment on vascular function in deoxycarticosterone acetate-salt hypertensive rats. Comparative study with verapamil. Planta Med. 2004; 70 (4): $334-41$.

18. Ajay M, Mustafa MR. Chronic treatment with flavonoids prevents endothelial dysfunction in spontaneously hypertensive rat aorta. J. Cardiovasc. Pharmacol. 2005; 46 (1): 36-40.

19. Ibarra M, Moreno L, Vera $\mathrm{R}$ et al. Effects of flavonoid quercetin and its methylated metabolite isorhamnetin in isolated arteries from spontaneously hypertensive rats. Planta Med. 2003; 69 (11): 995-1000.

20. Diederich D, Yang Z, Buhler FR et al. Impaired endothelium-dependent relaxations in hypertensive resistant arteries involve cyclooxygenase pathway. Am. J. Physiol. 1990; 258: H445-51.

21. Akpaffiong MJ, Taylor AA. Antihypertensive and vasodilator actions of antioxidants in spontaneously hypertensive rats. Am. J. Hypertens 1998; 11: 1450-60.
22. Neumann H, Carlsson K, Brom G. Uptake and localization of O(betahydroxyethyl) -rutosides in the venous wall, measured by laser scanning microscopy. Eur. J. Clin. Pharmacol. 1992; 43: 423-6.

23. Ruetten H, Zabel U, Linz W, Schmidt HH. Downregulation of soluble guanylyl cyclase in young and aging spontaneously hypertensive rats. Circ. Res. 1999; 85 (6): 534-41.

24. Morawietz H, Weber M, Rueckschloss U, Lauer N, Hacker A, Kojda G. Upregulation of vascular NAD (P) H oxidase subunit gp91phox and impairment of the nitric oxide signal transduction pathway in hypertension. Biochem. Biophysic. Res. Comm 2001; 285 : $1130-5$.

25. Kim DO, Lee KW, Lee HJ, Lee CY. Vitamin C equivalent antioxidant capacity (VCEAC) of phenolic phytochemicals. J. Agric. Food. Chem. 2002; 50 (13): 3713-17.

26. De Moura RS, Miranda DZ, Pinto AC et al. Mechanism of the endothelium-dependent vasodilation and the antihypertensive effect of Brazilian red wine. J. Cardiovasc. Pharmacol. 2004; 44: 302-9.

27. Woodman OL, Boujaoude M. Chronic treatment of male rats with diadzein and 17-beta-oestradiol induces the contribution of EDHF to endothelium-dependent relaxation. Br. J. Pharmacol. 2004; 141: $322-8$. 